\title{
Gut
}

Leading article

\section{A perspective on iron deficiency anaemia}

Patients with suspected iron deficiency anaemia account for some four per cent of the patients referred to our gastroenterology clinic. The first step in the investigation of these patients is to confirm the presence of iron deficiency. The next is to find out if the deficiency is a result of gastrointestinal blood loss or malabsorption. Menorrhagia is the major non-gastrointestinal cause of blood loss while dietary deficiency may be the sole cause or a contributing factor to the anaemia.

Microcytosis is also seen in patients with anaemia of chronic disease, the thalassaemia syndromes and, rarely, sideroblastic anaemia. A low serum ferritin or low serum iron with raised total iron binding capacity is usually sufficient to confirm iron deficiency. ${ }^{1}$ Occasionally these may be misleading. For example, inflammatory conditions such as inflammatory bowel disease or rheumatoid arthritis may lead to a spuriously high ferritin or low total iron binding capacity even in the presence of iron deficiency. In cases of doubt a bone marrow aspirate, which is the best guide to body iron stores, should be examined. ${ }^{2}$

\section{Colonic causes}

In the Western world, the most important cause of iron deficiency anaemia secondary to gastrointestinal blood loss is carcinoma of the colon. Different series have estimated it accounts for $4-13 \%$ of outpatients ${ }^{34}$ and $16 \%$ of inpatients. ${ }^{5}$ It is important not to miss this diagnosis because the disease is potentially fatal but can be cured if resected at an early stage. Most tumours presenting with iron deficiency anaemia are found on the right side of the colon. Although carcinoma of the colon is usually considered a disease of late middle age or elderly patients, it can occur in younger patients, particularly if associated with ulcerative colitis or a polyposis syndrome. Sporadic cases are seen in patients in their 20 s or early 30 s. ${ }^{6}$ In an adult population it cannot be excluded on the grounds of age alone. Bleeding adenomata can present with iron deficiency anaemia and should be resected.

Patients who are anti-coagulated and patients taking nonsteroidal anti-inflammatory drugs (NSAIDs) frequently become anaemic due to increased gastrointestinal blood loss. This anaemia is usually related to the presence of a lesion in the gastrointestinal tract, rather than to the drug itself. ${ }^{78}$ These patients should, therefore, be fully investigated to exclude the possibility of colonic cancer, in addition to stopping the drugs (where possible).

Angiodysplasia is commonly found in elderly patients with chronic gastrointestinal bleeding leading to iron deficiency.
In the absence of an obvious bleeding point, it is impossible to be certain that bleeding originates from the angiodysplasia until the rest of the gastrointestinal tract has been examined. An upper gastrointestinal endoscopy should be performed to exclude other abnormalities. Hereditary haemorrhagic telangiectasia often presents with iron deficiency anaemia although it can be usually diagnosed on clinical examination.

Anal lesions and haemorrhoids usually cause frank bleeding rather than anaemia while bleeding from diverticulosis is usually acute and massive. Anaemic patients with these lesions require full investigation as they are unlikely to be the cause of the anaemia even if frank rectal bleeding is present. ${ }^{9}$ Diverticulosis may complicate investigation of these patients, particularly if the colon is being assessed by barium enema examination as the diverticula may conceal the presence of adenomata or malignancy. ${ }^{10}$ " $\mathrm{Crohn's} \mathrm{disease} \mathrm{is}$ often associated with iron deficiency anaemia. Ulcerative colitis may present with anaemia when bowel symptoms are mild or ignored. Faecal occult blood studies have also uncovered previously unrecognised cases of ulcerative colitis. ${ }^{12}$ Low rectal or anal malignancy may be seen. Therefore sigmoidoscopy should be mandatory as part of the investigation of patients with iron deficiency anaemia.

\section{Upper gastrointestinal causes}

Oesophagogastroduodenoscopy in patients with anaemia often yields abnormal findings but it is not always clear if these abnormalities are the cause of the anaemia. A study of elderly hospital inpatients with iron deficiency anaemia found a high incidence (16\%) of dual pathology when both the upper and lower gastrointestinal tracts were examined. ${ }^{5}$ Although the incidence of dual pathology may be less in hospital outpatients populations $(9-14 \%),{ }^{313}$ it is recommended that the lower gastrointestinal tract should be examined in all patients presenting with anaemia, regardless of upper gastrointestinal endoscopy findings, except in those found to have malignancy of the upper gastrointestinal tract. ${ }^{4}$

Peptic ulceration may cause chronic gastrointestinal blood loss as well as an acute bleed. It is commonly asymptomatic in anaemic patients. ${ }^{14}$ NSAIDs may unmask an ulcer by causing bleeding. As NSAIDs may cause bleeding, however, throughout the gastrointestinal tract, unless the ulcer has stigmata of bleeding, the possibility of lesions elsewhere cannot be excluded. Studies with radiolabelled red cells and leucocytes suggest that, in $70 \%$ of patients with rheumatoid arthritis, NSAIDs are associated with inflammation and blood loss from the small intestine, especially in the region of 
the terminal ileum. ${ }^{15}$ NSAIDs may also trigger or exacerbate bleeding from vascular lesions in the intestine as well as malignant lesions. Antral ectasia (watermelon stomach) and carcinoma of the stomach ${ }^{16}$ have presented in this way. Severe erosive oesophagitis may be a cause of iron deficiency anaemia but hiatus hernia alone seems unlikely to cause iron deficiency anaemia.

\section{Small intestinal causes}

Bleeding from the small bowel may occur either from mucosal lesions or intramural lesions. Enteroscopy has identified three different types of mucosal lesion: red spot lesions with intact villi overlying them, erosions with damaged villi and discrete edges, and ulceration in which the thickness of the mucosa has been breached. ${ }^{17}$ Mural lesions include leiomyomata, carcinomata, small bowel lymphoma, secondary tumour deposits, and lipomata. Superior mesenteric angiography may also show these lesions and angiodysplasia.

\section{Malabsorption}

Inability to absorb iron also leads to anaemia. Iron deficiency may thus be found in postgastrectomy patients or patients with untreated coeliac disease. Most studies of patients presenting with anaemia find $2-3 \%$ of the population have villous atrophy. ${ }^{318}$ It is important to remember that coeliac disease can present at any age and that iron deficiency anaemia may be the only presenting symptom. ${ }^{19}$

\section{Recommended plan of investigation}

A recent study suggests that symptoms are an unreliable guide to the cause of anaemia in patients with gastrointestinal blood loss. ${ }^{3}$ Relevant aspects of the history include drug ingestion, particularly NSAIDs or anti-coagulants, a history of previous abdominal surgery such as gastrectomy, or of radiotherapy, or a family history of bowel cancer or colonic polyps. Dietary iron intake should be assessed. Although this is rarely the sole cause of the iron deficiency, in one study it was found to be a contributing factor in $57 \%$ of patients. ${ }^{3}$ While examining the patient, it is important to look for signs of systemic disease such as telangiectasia, signs of chronic liver disease or purpura. All patients being investigated for iron deficiency anaemia of gastrointestinal origin should have either rigid or flexible sigmoidoscopy (with a low threshold for biopsy). Routine blood tests of renal function and liver function and clotting studies are unhelpful in the investigation of patients with iron deficiency anaemia. ${ }^{3}$ They may be appropriate, however, in patients where the history or examination points to systemic disease such as chronic liver disease.

Ideally the initial investigation should be total colonoscopy as this will not only examine for malignant lesions but offers an opportunity to assess the mucosa for angiodysplasia. In addition lesions can be biopsied or cauterised..$^{20}$ At present, however, it still has limited availability so that for some patients sigmoidoscopy and barium enema examination provide an alternative.

Unless a bleeding point is seen on colonoscopy or the patient is found to have carcinoma of the colon, all patients should have upper gastrointestinal endoscopy. Variable proportions of patients with anaemia are found to have dual pathology $y^{3-5}$ and it is impossible to be certain which lesion is the major contributor to blood loss until the lesions have been treated. During gastroscopy, the folds of Kerckring are assessed for any reduction ${ }^{22}$ and distal duodenal biopsy specimens are taken in all patients for histological examination to look for villous atrophy.
Although few patients under investigation for iron deficiency anaemia will be found to have bleeding from the small intestine, these lesions can be difficult to find and therefore a disproportionate amount of time is spent in investigating these patients. Barium studies including small bowel enema are rarely helpful in this group of patients, ${ }^{423}$ although occasionally they may be useful in younger patients who are found to have Crohn's disease or a Meckel's diverticulum. Distal small bowel lesions may also be shown by reflux at barium enema or by entering the terminal ileum at colonoscopy.

In a young patient with a negative gastroscopy and large bowel study, a small bowel enema may be useful to exclude Crohn's disease. If there is suspicion of a Meckel's diverticulum an isotope scan may be helpful but it is important to remember that this investigation carries a significant proportion of false negatives. ${ }^{24}$ In older patients the small bowel enema is rarely helpful and the next investigation should be enteroscopy or mesenteric angiography. ${ }^{25}$ For patients who are bleeding acutely technetium-labelled red cell isotope scans or technetium-labelled sulphur colloid scans may provide additional information but when the rate of bleeding is slow, as in most patients with iron deficiency anaemia, these investigations are not helpful. ${ }^{26}$

For a few patients who experience severe or recurrent gastrointestinal bleeding of unknown origin, diagnostic laparotomy still has a place. ${ }^{27}$ The diagnostic yield at laparotomy can be improved by combining it with preoperative or peroperative angiography to identify the bleeding segment of bowel and with on table endoscopy to find the lesion. ${ }^{28}$

\section{Outcome}

Initial investigations including colonoscopy or sigmoidoscopy and barium enema and upper gastrointestinal endoscopy with duodenal biopsy will establish a cause for the anaemia in $80 \%$ of patients. Enteroscopy has identified small intestinal lesions in a further $30-40 \%$ of patients in whom the first line investigations were normal. Where there is a possibility that the anaemia may be exacerbated by drugs such as NSAIDs or anti-coagulants, these should be stopped if possible. For patients with vascular abnormalities endoscopic cautery may be appropriate. If the lesions are restricted, however, to a small segment of bowel and the patient suffers from recurrent episodes of anaemia, then surgical resection is appropriate. Other treatments that may be useful for patients with recurrent anaemia from vascular abnormalities include oestrogens, ${ }^{29}$ ethamsylate, and tranexamic acid.

For the remaining $20 \%$ of patients in whom no cause for the anaemia has been found, careful follow up with monitoring of haemoglobin and red cell indices is required. If the anaemia persists or frank bleeding occurs, further investigation should be done. A recent follow up study by Sahay and Scott, ${ }^{30}$ however, suggests that in two thirds of these patients the anaemia resolves and does not recur. The extent to which the rest of the patients should be investigated further depends on their age and general health, symptoms related to the anaemia, and the need for continuing iron treatment or blood transfusions, where antibody formation may cause major problems.

\section{Prospects}

Earlier detection of carcinoma of the bowel by the use of faecal occult blood screening tests should reduce the number of these patients presenting with iron deficiency anaemia. A negative test in an anaemic patient cannot exclude the possibility of carcinoma of the colon and therefore patients 
with iron deficiency anaemia should be investigated for this regardless of the presence or absence of frank or occult faecal bleeding. An alternative approach is to offer a single examination by flexible sigmoidoscopy to the whole population at age $55 .{ }^{31}$ In high risk populations such as those with a previous adenoma, the intervals between repeat colonscopy have been extended from one to three years. ${ }^{32}$

There is increasing awareness of the contribution of NSAIDs to gastrointestinal blood loss. Nabumetone is an attempt to overcome direct contact between the drug and the small intestinal mucosa as it is ingested as a prodrug and metabolised to the active drug after absorption. Misoprostol ${ }^{33}$ or $\mathrm{H}_{2}$ receptor antagonists ${ }^{34}$ may represent an alternative approach as, when used in combination with NSAIDs, they provide some protection against gastric and duodenal damage. No longterm data are available, however, as to whether this approach will reduce the incidence of gastrointestinal bleeding.

J M SAYER

Medical Research Centre,

City Hospital,

Nottingham NG5 IPB

1 Massey AC. Microcytic anaemia. Differential diagnosis and management of iron deficiency anaemia. Med Clin N Am 1992; 76: 549-66.

2 Doube A, Davis M, Smith JG, Maddison PJ, Collins AJ. Structured approach to the investigation of anaemia in patients with rheumatoid arthritis. Ann Rheum Dis 1992; 51: 469-72.

3 McIntyre AS, Long RG. Prospective survey of investigations in outpatients referred with iron deficiency anaemia. Gut 1993; 34: 1102-7.

4 Till SH, Grundman MJ. A prospective audit of patients presenting with iron deficiency anaemia and faecal occult blood loss [Abstract]. Gut 1992; 33 (suppl): S31.

5 Cook IJ, Pavli P, Riley JW, Goulston KJ, Dent OF. Gastrointestinal investigation of iron deficiency. $B M \mathcal{J} 1986 ; 292$ : $1380-2$

6 O'Reilly D, Long RG. Carcinoma of the colon presenting with dysplasia. Postgrad Med F 1987; 63: 215-6.

7 Jaffin BW, Bliss CM, Lamont JT. Significance of occult gastrointestinal bleeding during anticoagulation therapy. Am f Med 1987; 83: 269-72.

8 Pye G, Ballantyne KC, Armitage NC, Hardcastle JD. Influence of nonsteroidal anti-inflammatory drugs on the outcome of faecal occult blood tests in screening for colorectal cancer. BMF 1987; 294: 1510-1.

9 Goulston KJ, Cook I, Dento F. How important is rectal bleeding in the diagnosis of bowel cancer and polyps? Lancet 1986; ii: 261-4.

10 Aldridge MC, Sim AJW. Colonoscopy findings in symptomatic patients without X ray evidence of colonic neoplasms. Lancet 1986; ii: 833-4.
11 Boulos PB, Karamanolis DG, Salmon PR, Clark CG. Is colonoscopy necessary in diverticular disease? Lancet 1984; i: 95-6.

12 Rosenthal P, Jennings MT. Comparison of fecal occult blood tests for detection of gastrointestinal bleeding in pediatric patients. Am $\mathcal{F}$ Gastroenterol 1992; 87: $1575-9$.

13 Zuckerman G, Benitez J. A prospective study of bidirectional endoscopy (colonoscopy and upper endoscopy) in the evaluation of patients with occult gastrointestinal bleeding. Am F Gastroenterol 1992; 87: 62-6.

14 Arnold MM, McKenna K. NSAID associated gastroduodenal damage - do duodenal ulcers cause more symptoms and complications? $\mathrm{Br} \mathcal{F}$ Rheumato 1993; 32 (suppl): 109.

15 Bjarnason I, Zanelli G, Pouse P, Smethurst P, Smith T, Levi S, et al. Blood and protein loss via small-intestinal inflammation induced by non-steroidal anti-inflammatory drugs. Lancet 1987; ii: 711-4.

16 Moreno-Otero R, Rodriguez S, Carbo J, Mearin F, Pajares JM. Acute upper gastrointestinal bleeding as primary symptom of gastric carcinoma. F Surg Oncol 1987; 36: 130-3.

17 Morris AJ, Wasson LA, MacKenzie JF. Small bowel enteroscopy in undiagnosed gastrointestinal blood loss. Gut 1992; 33: 887-9.

18 Tobin MV, Gilmore IT. Gastrointestinal investigation of iron deficiency anaemia. BMF 1986; 292: 1738 .

19 Depla ACTM, Bartelsman JFWM, Mulder CJJ, Tytgat GNJ. Anaemia: monosymptomatic celiac disease. Hepatogastroenterology 1990; 37: 90-1.

20 Lindsay DC, Freeman JG, Cobden I, Record CO. Should colonoscopy be the first investigation for colonic disease? $B M \mathcal{F} 1988 ; 296$ : 167-9.

21 Durdey P, Weston PMT, Williams NS. Colonoscopy or barium enema as initia investigation of colonic disease. Lancet 1987; ii: 549-51.

22 McIntyre AS, Ng DPK, Smith JA, Amoah JA, Long RG. The endoscopic appearance of duodenal folds is predictive of untreated celiac disease. Gastrointest Endosc 1992; 38: 148-51.

23 Rockey DC, Cello JP. Gastrointestinal evaluation of chronic iron deficiency anaemia. Gastroenterology 1992; 102: A24.

24 Sfakianakis GN, Conway JJ. Detection of ectopic gastric mucosa in Meckel's diverticulum and in other aberrations by scintigraphy: I Pathophysiology and 10-year clinical experience. $\mathcal{F}$ Nucl Med 1981;22:647-54

25 Steger AC, Spencer J. Obscure gastrointestinal bleeding. BMF 1988; 296: 3. 6 Baulieu F, Baulieu JL, Valmalle R, Secchi V, Pottier JM, Dorval E, et al. Localisation scintigraphique des saignements digestifs. Ann Chir 1992; 46 : 319-23.

27 Thompson JN, Salem RR, Hemingway AP, Rees HC, Hodgson HJF, Wood $\mathrm{CB}$, et al. Specialist investigation of obscure gastrointestinal bleeding. Gut 1987; 28: 47-51.

28 Lau WY, Fan ST, Wong SH, Wong KP, Poon GP, Chu KW, et al. Preoperative and intraoperative localisation of gastrointestinal bleeding of obscure origin. Gut 1987; 28: 869-77.

29 Van Cutsem E, Rutgeerts P, Vantrappen G. Treatment of bleeding gastrointestinal vascular malformations with oestrogen-progesterone. Lancet 1990 335: 953-5.

30 Sahay R, Scott BB. Iron deficiency anaemia - how far to investigate? Gut 1993; 34: $1427-8$.

31 Atkin WS, Cuzick J, Northover JMA, Whynes DK. Prevention of colorecta cancer by once-only sigmoidoscopy. Lancet 1993; 341: 736-40.

32 Ahlquist DA, McGill DB, Schwartz S, Taylor WF, Owen RA. Fecal blood levels in health and disease. $N$ Engl F Med 1985; 312: 1422-8.

33 Fenn GC, Robinson GC. Misoprostol a logical therapeutic approach to gastro duodenal mucosal injury induced by non steroidal anti-inflammatory drugs? $\exists$ Clin Pharm Ther 1991; 16: 385-409.

34 Hudson N, Taha AS, Sturrock RD, Russell RI, Hawkey CI. Famotidine in healing of nonsteroidal anti-inflammatory drug associated gastroduodenal ulceration. Gastroenterology 1993; 104: A106. 